\title{
Rectraction
}

Yaswant Kumar Pankaj^, Mahesh Jagadale Vasantrao, Nilmani Prakash, Raj Kumar Jat, Rajesh Kumar, Vinay Kumar, Pankaj Kumar

\section{Characterization of wheat (Triticum aestivum L.) genotypes unraveled by molecular markers considering heat stress}

https://doi.org/10.1515/opag-2019-0037

DOI: 10.1515/opag-2019- 0004

Retraction of: Yaswant Kumar Pankaj, Mahesh Jagadale Vasantrao, Nilmani Prakash, Raj Kumar Jat, Rajesh Kumar, Vinay Kumar, Pankaj Kumar, Characterization of wheat (Triticum aestivum L.) genotypes unraveled by molecular markers considering heat stress, Open Agriculture, 2019, Vol 4, 1, 41-51

The article to be withdrawn due to the conflict of interests between authors.

*Corresponding author:Yaswant Kumar Pankaj, Department of Agricultural Biotechnology and Molecular Biology, Dr. RPCAU, Pusa, Bihar, India, 848125, E-mail: yaswantkumar19994@gmail.com Mahesh Jagadale Vasantrao, Department of Agricultural Biotechnology and Molecular Biology, Dr. RPCAU, Pusa, Bihar, India, 848125 Nilmani Prakash, Raj Kumar Jat, Borlaugh Institute of South Asia, Pusa, Bihar, India, 848125

Rajesh Kumar, Department of Plant Breeding and Genetics, Dr. RPCAU, Pusa, Bihar, India, 848125

Vinay Kumar, Indian Agricultural Research Institute, Regional Station, Pusa, Bihar, India, 848125

Pankaj Kumar, Cereal Systems Initiative for South Asia, CIMMYT,

Patna, Bihar, India, 803212 